\title{
DETERMINING COMPRESSION FACTOR OF QUASILOGARITHMIC QUANTIZERS FOR LAPLACIAN SOURCE IN NARROW DYNAMIC VARIANCE RANGE
}

\author{
UDC (621.391:004):517.225
}

\section{Milan Tančić, Zoran Perić, Aleksandra Jovanović, Stefan Tomić}

University of Niš, Faculty of Electronic Engineering, Department of Telecommunications, Niš, Republic of Serbia

\begin{abstract}
In this paper, it has been performed an optimization of compression factor of quasi-logarithmic quantizer for the case when a signal with Laplacian probability density function is brought on the input of quantizer. There has been proposed a new two-step method for determination of optimal compression factor in terms of the mean-square error (MSE) distortion. Two different manners for compression factor optimization have also been considered, by using the Muller's iterative method and the new two-step method. Emphasis is placed on locating slightly less accurate but much simpler solution, by comparing the Muller's iterative method and the new two-step method. Analysis of procedures is described in detail.
\end{abstract}

Key words: companding quantizer, Laplacian probability density function, Muller's iterative method, new two-step optimization method

\section{INTRODUCTION}

Quantization process can be performed using uniform and non-uniform quantizers. Uniform quantizers are suitable for signals with uniform distribution (uniform probability density function) due its equidistant decision intervals and representational levels. Unlike uniform quantizers, the non-uniform quantizers have smaller decision intervals where probability of input signal is high and larger decision intervals where probability of input signal is low [1], [2], [3]. Speech signal has non-uniform distribution and because of it, it is appropriate to use non-uniform quantizers for signal processing. Non-uniform quantizers can be designed as Lloyd-Max and companding quantizers.

Companding concept is useful in analysis of the non-uniform quantizers with a large number of quantization levels. Companding quantizers can be optimal and logarithmic,

Received May 25, 2016

Corresponding author: Milan Tančić

University of Niš, Faculty of Electronic Engineering, Department of Telecommunications, Republic of Serbia

E-mail: milan_tancic@yahoo.com 
and within the logarithmic there are $A$ and $\mu$-logarithmic quantizers. In this paper, we assume that input signal is with Laplacian probability density function and pay attention on $\mu$-logarithmic companding quantizer which provides almost constant signal to quantization noise ratio (SQNR) in a wide range of input variance. A quasi-logarithmic companding quantizer is suitable when the input signal's variance changes with the time in a wide range, as it is the case with the speech signals [1].

The emphasis in this paper is placed on finding the optimal value of compression factor by using two methods. A new two-step method is introduced to determine the optimal value of compression factor. Thus obtained optimal compression factor is compared with the result achieved with the Muller's iterative method. The behavior of SQNR is also analyzed in a narrow range of input signal variance. The goal of this paper is formulation of a simple method for compression factor optimization which provides results comparable to those obtained using the Muller's iterative method. Both methods start from the first derivate of distortion which is obtained by using the Bennett's integral, and setting it to zero. After that we apply the Muller's iterative method and new two-step method and discuss results. These optimization methods can be also applied to the image and video processing [4], [5].

The Muller's iterative method works on the principle of approximating the function in the vicinity of the roots of the quadratic polynomial. For solving the obtained transcendental equation by using the new two-step method we notice that a substitution which reduces complexity of equation into a seeming quadratic equation can be introduced. We determine the solution of quadratic equation, replace the introduced substitution and obtain a simple equation for determining the optimal value of compression factor $\mu$.

One of the parameters for both methods is the support range of quantizer and it has to be correctly determined due to its influence on the SQNR [2], [3], [6], [7] and [8]. The region of an input signal is divided by the scalar quantization procedure into a granular region and an overload region, which are separated by the support region thresholds $-x_{\max }$ and $x_{\max }$. These thresholds define support region for quantizer [1], [2], [3], [6] and [7]. The support region has great influence on the total distortion, because the granular and overload distortion compose total distortion. Wider support region provides smaller overload distortion, but larger granular distortion while narrower support region provides smaller granular but greater overload distortion, and because of that fact this value has to be optimal too. Equation for finding optimal support limit value of quasi-logarithmic quantizer is derived in paper [6] and that closed-form formula is used in this analysis.

The rest of the paper is organized as follow. In Section 2 a description of companding quantizer for a Laplacian source is given. Also the Muller's iterative method and the new two-step method are described in detail. In Section 3 numerical results are shown and analysis of these results is given. In Section 4 the contributions of the paper are summarized.

\section{COMPRESSION FACTOR DETERMINATION FOR QUASI-LOGARITHMIC QUANTIZER}

Quantization process represents mapping $Q\left(\left[x_{i-1}, x_{i}\right) \rightarrow y_{i}, i=1, \ldots, N\right.$, where $N$ is the number of quantizer levels, $x_{i}, i=0, \ldots, N$, are decision thresholds, and $y_{i}, i=1, \ldots, N$, are representation levels [1]. For signals with unlimited amplitude can be taken $x_{0} \rightarrow-\infty$ and $x_{N} \rightarrow+\infty$. Segment $\left[x_{i-1}, x_{i}\right)$ is noted as $\Delta_{i}, i=1, \ldots, N$. Segments $\Delta_{2}, \Delta_{3}, \ldots, \Delta_{N-1}$ that are limited from both sides present granular region. The first and the last segments $\Delta_{1}$ and $\Delta_{N}$ are 
unlimited from one side and they make overload region. During the quantization process the irreversible error is made due rounding current values of input signal on representation level. This error is called quantization error. Quantization error can be expressed by the size called distortion which represents average value of mean-square error.

Distortion presents the sum of two parts: granular and overload distortion. In this paper we use Bennett's integral to obtain granular distortion (1) and expression (2) for overload distortion [1]:

$$
\begin{gathered}
D_{g}=\frac{x_{\max }^{2}}{3 N^{2}} \int_{-x_{\max }}^{x_{\max }} \frac{p(x)}{\left[c^{\prime}(x)\right]^{2}} d x, \\
D_{o}=2 \int_{x_{N}}^{+\infty}\left(x-y_{N}\right)^{2} p(x) d x,
\end{gathered}
$$

where $c^{\prime}(x)$ presents the first derivative of compressor function used in quasi-logarithmic quantizer, $p(x)$ is a Laplacian probability density function, $N$ is the number of quantization levels while $x_{N}=x_{\max }$ presents the support range of quantizer.

In this paper, Laplacian probability density function is used and is defined as:

$$
p(x)=\frac{1}{\sqrt{2} \sigma} \exp \left(-\frac{|x| \sqrt{2}}{\sigma}\right),
$$

where $\sigma$ presents standard deviation of the random variable $x$. $\mu$-law compression function, on which quantizer's design is based, is defined with [1], [6], [7] and [8]:

$$
c(x)=\frac{x_{\max }}{\ln (1+\mu)} \ln \left(1+\mu \frac{|x|}{x_{\max }}\right) \operatorname{sgn}(x),|x| \leq x_{\max },
$$

where $x_{\max }$ is support range of quasi-logarithmic quantizer, whereas $\mu$ is compression factor [1].

By substituting (3) and (4) in (1) and introducing approximation $y_{N} \approx x_{\max }$, we obtain the following variance depended expression for total distortion [2], [3]:

$$
D=\frac{\ln ^{2}(1+\mu) \sigma^{2}}{3 N^{2}}\left(\frac{x_{\max }^{2}}{\mu^{2} \sigma^{2}}+\frac{\sqrt{2} x_{\max }}{\mu \sigma}+1\right)+\sigma^{2} \exp \left(-\frac{\sqrt{2} x_{\max }}{\sigma}\right) .
$$

As previously mentioned, total distortion presents the sum of granular and overload distortion: $D(Q)=D_{g}(Q)+D_{o}(Q)$. In this paper we design companding quantizer for unit variance $\sigma^{2}=1$, as well as for narrow range of input signal variances, so that the expression for total distortion becomes:

$$
D=\frac{\ln ^{2}(1+\mu)}{3 N^{2}}\left(\frac{x_{\max }^{2}}{\mu^{2}}+\frac{\sqrt{2} x_{\max }}{\mu}+1\right)+\exp \left(-\sqrt{2} x_{\max }\right) .
$$

Expression (5) is very important for our work because it represents the starting point for both methods. In both methods it is required that the first derivate of distortion with respect to compression factor $\mu$ which equates to zero. 
For determining optimal value for compression factor of the considered quasilogarithmic companding quantizer, we need to know optimal support region threshold and for this reason we use, in both methods, the closed-form formula from [6]:

$$
x_{\max }=\frac{1}{\sqrt{2}} \ln \left(\frac{3 \mu N^{2}}{\ln ^{2}(\mu+1)}\right) .
$$

The basis of Muller's is approximation of function in the neighborhood of the root by a quadratic polynomial [9]. In fact, Muller's method generalizes the secant method of root finding by using quadratic 3-point interpolation [10], [11]. It uses three points, then constructs parabola trough these three points and takes the intersection of this parabola with $x$-axis to be the next approximation [9].

For the new two-step method, it is also necessary to equalize the first derivative of total distortion expression (5) in respect to compression factor $\mu$ with zero, and we obtain the following equation:

$$
\mu^{2}+\sqrt{2} x_{\max }\left(1-\frac{\mu+1}{\mu} \frac{\ln (\mu+1)}{2}\right) \mu+x_{\max }^{2}\left(1-\frac{\mu+1}{\mu} \ln (\mu+1)\right)=0 .
$$

By observing equation (8) we concluded that a substitution can be introduced:

$$
t_{0}=\frac{\mu+1}{\mu} \ln (\mu+1) \text {. }
$$

By introducing the substitution (9), transcendent equation (8) has been reduced to simple quadratic equation where compression factor $\mu$ is variable after which equation can be solved:

$$
\mu^{2}+\sqrt{2} x_{\max }\left(1-\frac{t_{0}}{2}\right) \mu+x_{\max }^{2}\left(1-t_{0}\right)=0
$$

which gives two solutions:

$$
\mu=\frac{\sqrt{2} x_{\max }}{4}\left[\left(t_{0}-2\right) \pm \sqrt{t_{0}{ }^{2}+t_{0}-1}\right],
$$

but it is considered only one with physical sense, that is solution that gives positive values for compression factor.

The optimal value of the compression factor is obtained for the case when the distortion has a minimum value which provides the highest SQNR. The second step of this method enables us to satisfy this condition. For that purpose we apply two closed-form equations for the first and second step of the method:

$$
\begin{aligned}
& \mu_{1}=\frac{\sqrt{2} x_{\max }}{4}\left[\left(\left(\frac{\mu_{0}+1}{\mu_{0}} \ln \left(\mu_{0}+1\right)\right)-2\right)+\right. \\
& \sqrt{\left.\left(\frac{\mu_{0}+1}{\mu_{0}} \ln \left(\mu_{0}+1\right)\right)^{2}+\left(\frac{\mu_{0}+1}{\mu_{0}} \ln \left(\mu_{0}+1\right)\right)-1\right]},
\end{aligned}
$$

for first step of the new two-step method, and: 


$$
\begin{aligned}
& \mu_{2}=\frac{\sqrt{2} x_{\max }}{4}\left[\left(\left(\frac{\mu_{1}+1}{\mu_{1}} \ln \left(\mu_{1}+1\right)\right)-2\right)+\right. \\
& \sqrt{\left.\left(\frac{\mu_{1}+1}{\mu_{1}} \ln \left(\mu_{1}+1\right)\right)^{2}+\left(\frac{\mu_{1}+1}{\mu_{1}} \ln \left(\mu_{1}+1\right)\right)-1\right]},
\end{aligned}
$$

for the second step of the method. In expressions (12) and (13) it can be observed that support range of quasi-logarithmic quantizer is an important parameter for compression factor optimization. In this purpose, support range value must be calculated for every step, which means that we calculate support range, distortion and SQNR for starting value of compression factor. After that we obtain compression factor value for the first step and calculate all of these values for this step. The procedure is the same for second step of this two-step method for compression factor optimization.

Quality of quantized signal is usually expressed through signal-to-quantization noise ratio which is defined as:

$$
\mathrm{SQNR}[\mathrm{dB}]=10 \log _{10}\left(\frac{\sigma^{2}}{D}\right)
$$

Average value of SQNR, by which we can estimate the optimal compression factor value in narrow range of input signal variances, is calculated using following expression:

$$
\mathrm{SQNR}_{\text {average }}[\mathrm{dB}]=\frac{\sum_{i=1}^{M} \mathrm{SQNR}[\mathrm{dB}]}{M} .
$$

where $M$ presents the number of samples in the range of variances.

\section{NUMERICAL RESULTS AND ANALYSIS}

In this section the numerical results obtained during the process of compression factor optimization by using two different methods are presented. For the case when a signal with Laplacian probability density function of unit variance is brought on the input of companding quantizer, the compression factor and support limit of quantizer are calculated in two different ways, using Muller's iterative method and presented two-step method. The results are shown in tables and compared, after which we derive certain conclusions.

In tables the results of compression factor optimization $\mu$ are shown for two methods. Tables 1, 2, 3 and 4 show values for compression factor $\mu$, distortion $D$ and signal to quantization noise ratio SQNR during optimization process by using the new two-step method for different numbers of quantization levels $N$.

At first, we need to have a starting value for compression factor and for this analysis it is used $\mu=128$, corresponding to approximately half of compression factor value in ITU-T recommendation G.711. Due to the importance of support range value, expression (7) has been used to obtain support range value that is calculated in both steps of the new two-step method, but also in initial step for starting value of compression factor. After that, in the first step the compression factor value has been calculated by using (12), support region value 
using expression (7), while the distortion $D$ is obtained by using expression (6) and in the end SQNR by using (14). After that, in the second step expression (13) has been used for calculating $\mu$ value that presents the optimal value, expression (6) for distortion $D$ in this step and expression (14) for final signal-to-quantization noise-ratio SQNR.

Table 1 Compression factor $\mu$, distortion $D$, and SQNR of the new two-step method for $N=32$

\begin{tabular}{ccccc}
\hline Step & $\mu$ & $x_{\max }$ & $D$ & SQNR[dB] \\
\hline$\mu_{0}$ & 128 & 6.872 & 0.00835415 & 20.781 \\
$\mu_{1}$ & 19.811 & 6.218 & 0.00477807 & 23.2075 \\
$\mu_{2}$ & 10.311 & 6.073 & 0.00436196 & 23.6032 \\
\hline
\end{tabular}

Table 2 Compression factor $\mu$, distortion $D$, and SQNR of the new two-step method for $N=64$

\begin{tabular}{ccccc}
\hline Step & $\mu$ & $x_{\max }$ & $D$ & SQNR[dB] \\
\hline$\mu_{0}$ & 128 & 7.85223 & 0.00211105 & 26.755 \\
$\mu_{1}$ & 22.6374 & 7.23476 & 0.00130122 & 28.8565 \\
$\mu_{2}$ & 12.5906 & 7.09199 & 0.00121541 & 29.1528 \\
\hline
\end{tabular}

Table 3 Compression factor $\mu$, distortion $D$, and SQNR of the new two-step method for $N=128$

\begin{tabular}{ccccc}
\hline Step & $\mu$ & $x_{\max }$ & $D$ & SQNR[dB] \\
\hline$\mu_{0}$ & 128 & 8.83234 & 0.000533447 & 32.7291 \\
$\mu_{1}$ & 25.4629 & 8.24843 & 0.000349829 & 34.5614 \\
$\mu_{2}$ & 14.9603 & 8.1095 & 0.00033213 & 34.7869 \\
\hline
\end{tabular}

Table 4 Compression factor $\mu$, distortion $D$, and SQNR of the new two-step method for $N=256$

\begin{tabular}{ccccc}
\hline Step & $\mu$ & $x_{\max }$ & $D$ & SQNR[dB] \\
\hline$\mu_{0}$ & 128 & 9.81245 & 0.000134797 & 38.7032 \\
$\mu_{1}$ & 28.2885 & 9.25981 & 0.000093138 & 40.3087 \\
$\mu_{2}$ & 17.4093 & 9.12575 & 0.000089491 & 40.4822 \\
\hline
\end{tabular}

Tables 5, 6, 7 and 8 show compression factor changing during optimization process using the Muller's iterative method for different values of quantization levels. For using Miller's method we have to set the initial values for the compression factor, and it is done by setting $\left(x_{1}, x_{2}, x_{3}\right)=(1,255,128)$, where $\left(x_{1}, x_{2}\right)$ represent border values. After that we need to calculate parameters for the Muller's method [9] in order to obtain the optimal value of compression factor during iterations. For every iteration the relative error is calculated and based on that we stop algorithm when error is smaller than predefined threshold $\varepsilon$, which is $\varepsilon=1 \%$. Starting with the real numbers for border points $\left(x_{1}, x_{2}\right)=(1$, $255)$, we ensure that the algorithm converges to the solution, which is thus certainly to be the real value. The optimization process of compression factor using Muller's iterative method is performed for the same quantization level numbers as the new two-step method. 
Table 5 Parameters for analysis of the accuracy of Muller's iterative method for $N=32$

\begin{tabular}{cccccc}
\hline $\mathrm{I}$ & $x_{1}$ & \multicolumn{1}{c}{$x_{2}$} & \multicolumn{1}{c}{$x_{3}$} & \multicolumn{1}{c}{$x_{4}$} & $\delta[\%]$ \\
\hline 1 & 1 & 255 & 128 & 88.0280 & 45.4 \\
2 & 1 & 128 & 88.0280 & 54.8561 & 60.47 \\
3 & 1 & 88.0280 & 54.8561 & 36.2045 & 51.52 \\
4 & 1 & 54.8561 & 36.2045 & 24.1396 & 49.98 \\
5 & 1 & 6.2045 & 24.1396 & 16.8432 & 43.31 \\
6 & 1 & 24.1396 & 16.8432 & 12.4921 & 34.83 \\
7 & 1 & 16.8432 & 12.4921 & 10.1286 & 23.34 \\
8 & 1 & 12.4921 & 10.1286 & 9.0669 & 11.62 \\
9 & 1 & 10.1286 & 9.0669 & 8.7428 & 3.71 \\
10 & 1 & 9.0669 & 8.7428 & 8.6904 & 0.6 \\
\hline
\end{tabular}

Table 6 Parameters for analysis of the accuracy of Muller's iterative method for $N=64$

\begin{tabular}{clllll}
\hline $\mathrm{I}$ & $x_{1}$ & \multicolumn{1}{c}{$x_{2}$} & \multicolumn{1}{c}{$x_{3}$} & $x_{4}$ & $\delta[\%]$ \\
\hline 1 & 1 & 255 & 128 & 88.5680 & 44.52 \\
2 & 1 & 128 & 88.5680 & 55.6518 & 59.14 \\
3 & 1 & 88.5680 & 55.6518 & 37.2201 & 49.52 \\
4 & 1 & 55.6518 & 37.2201 & 25.3594 & 46.77 \\
5 & 1 & 37.2201 & 25.3594 & 18.3005 & 38.57 \\
6 & 1 & 25.3594 & 18.3005 & 14.2467 & 28.45 \\
7 & 1 & 18.3005 & 14.2467 & 12.2252 & 16.53 \\
8 & 1 & 14.2467 & 12.2252 & 11.4630 & 6.64 \\
9 & 1 & 12.2252 & 11.4630 & 11.2913 & 1.52 \\
10 & 1 & 11.4630 & 11.2913 & 11.2726 & 0.16 \\
\hline
\end{tabular}

Table 7 Parameters for analysis of the accuracy of Muller's iterative method for $N=128$

\begin{tabular}{ccllcc}
\hline I & $x_{1}$ & \multicolumn{1}{c}{$x_{2}$} & \multicolumn{1}{c}{$x_{3}$} & \multicolumn{1}{c}{$x_{4}$} & $\delta[\%]$ \\
\hline 1 & 1 & 255 & 128 & 89.1369 & 43.59 \\
2 & 1 & 128 & 89.1369 & 56.5070 & 57.74 \\
3 & 1 & 89.1369 & 56.5070 & 38.3309 & 47.42 \\
4 & 1 & 56.5070 & 38.3309 & 26.7210 & 43.44 \\
5 & 1 & 38.3309 & 26.7210 & 19.9559 & 33.90 \\
6 & 1 & 26.7210 & 19.9559 & 16.2559 & 22.76 \\
7 & 1 & 19.9559 & 16.2559 & 14.5981 & 11.35 \\
8 & 1 & 16.2559 & 14.5981 & 14.0895 & 3.61 \\
9 & 1 & 14.5981 & 14.0895 & 14.0065 & 0.59 \\
10 & 1 & 14.0895 & 14.0065 & 14.0001 & 0.045 \\
\hline
\end{tabular}

Table 8 Parameters for analysis of the accuracy of Muller's iterative method for $N=256$

\begin{tabular}{clllll}
\hline $\mathrm{I}$ & $x_{1}$ & \multicolumn{1}{c}{$x_{2}$} & \multicolumn{1}{c}{$x_{3}$} & $x_{4}$ & $\delta[\%]$ \\
\hline 1 & 1 & 255 & 128 & 89.7358 & 45.69 \\
2 & 1 & 128 & 89.7358 & 57.4240 & 56.27 \\
3 & 1 & 89.7358 & 57.4240 & 39.5407 & 45.22 \\
4 & 1 & 57.4240 & 39.5407 & 28.2291 & 40.07 \\
5 & 1 & 39.5407 & 28.2291 & 21.8116 & 29.42 \\
6 & 1 & 28.2291 & 21.8116 & 18.5076 & 17.85 \\
7 & 1 & 21.8116 & 18.5076 & 17.2046 & 7.57 \\
8 & 1 & 18.5076 & 17.2046 & 16.8868 & 1.88 \\
9 & 1 & 17.2046 & 16.8868 & 16.8491 & 0.22 \\
10 & 1 & 16.8868 & 16.8491 & 16.8469 & 0.01 \\
\hline
\end{tabular}


After determining the optimal value of compression factor $\mu$ for quasi-logarithmic quantizer in the case when Laplacian source at the quantizer input has unit variance, the optimal value of compression factor will be searched for in a narrow range of input signal variance, $\sigma^{2} \in[-3 \mathrm{~dB}, 3 \mathrm{~dB}]$. In Fig. 1 , for $N=256$, an analysis of obtaining the optimal compression factor $\mu$ is presented. We start from the optimal value $x_{\max }$ for unit variance and analyze which value of compression factor is optimal for constant value of $x_{\max }=9.12$. The analysis is made for values of $\mu(\mu=14,18,28,40,70)$, which are from the wide range of compression factor, and calculated the average value of SQNR in the order to conclude which value of compression factor $\mu$ is optimal for narrow range of input signal variance.

From Fig. 1 and by calculating the average values of SQNR for each compression factor value, it can be seen that for the narrow range of input signal variance $[-3 \mathrm{~dB}, 3 \mathrm{~dB}]$ the optimal value of compression factor is $\mu=18$ for $N=256$, which is the closest value to the optimal value for unit variance $\mu=17.4$ according to the new two-step method. In this range of input signal variance the average values of SQNR for $\mu=14,18,28,40,70$ is $\mathrm{SQNR}=39.7067 \mathrm{~dB}, 39.7514 \mathrm{~dB}, 39.6479 \mathrm{~dB}, 39.4270 \mathrm{~dB}, 38.9137 \mathrm{~dB}$, respectively from which it can be observed that the average value of SQNR for $\mu=18$ is better than the values of average SQNR for the rest of analyzed values of $\mu$.

From Fig. 1 it can be seen that in the zone of small input variance $\left(\sigma^{2}=-3 \mathrm{~dB}\right)$ SQNR increases until compression factor reaches value of $\mu=28$ and after that SQNR begins to decrease along with its maximum.

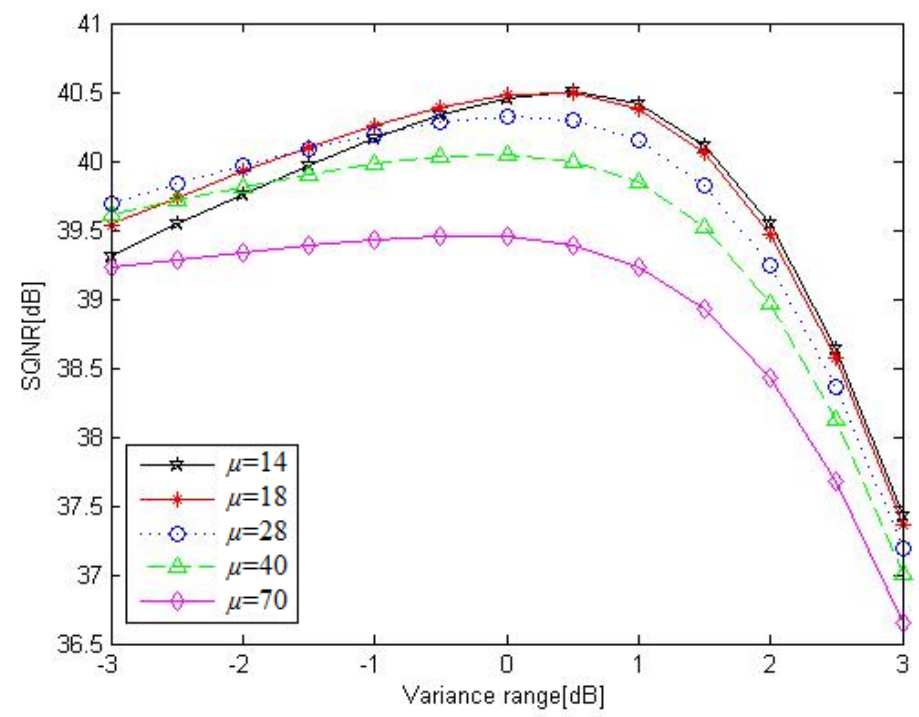

Fig. 1 SQNR over variance range of input signal [-3dB, 3dB] for $N=256, x_{\max }=9.12$ and different values of compression factor $\mu$

Further increasing of the compression factor decreases the value of SQNR, the mean value of SQNR and its maximum value. In order to satisfy the condition that on both sides of the range approximately the same SQNR have to be, we must consider the range of the input variance $\sigma^{2} \in[-4 \mathrm{~dB}, 2 \mathrm{~dB}]$. 


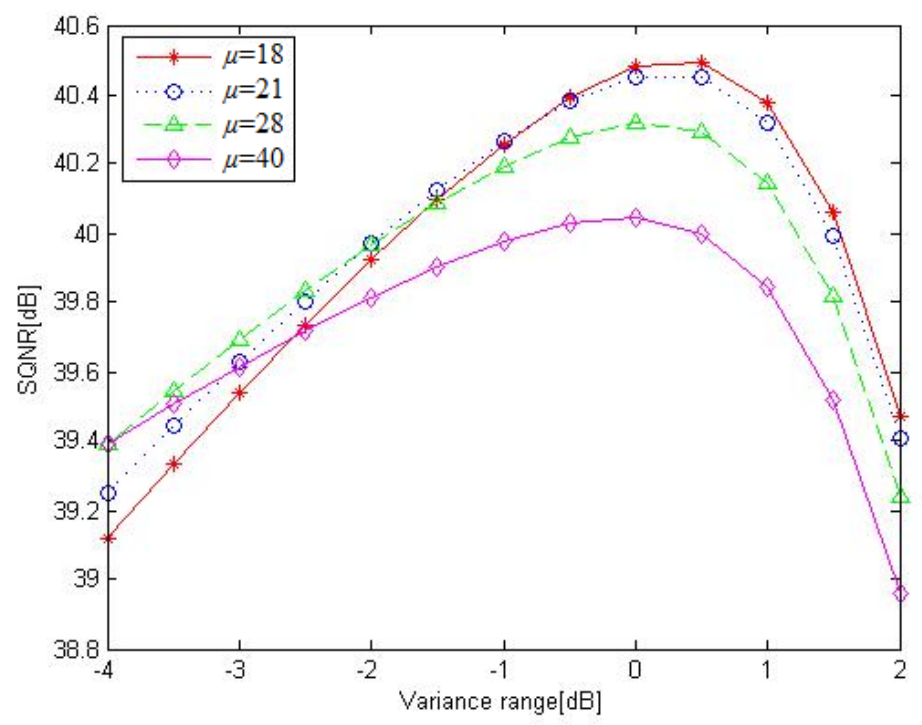

Fig. 2 SQNR over variance range of input signal [-4dB, 2dB] for $N=256, x_{\max }=9.12$ and different values of $\mu$

From Fig. 2 and by calculating the average values of SQNR for the following values of compression factor $\mu=18,21,28,40$, it can be seen that for the narrow range of input signal variance $[-4 \mathrm{~dB}, 2 \mathrm{~dB}]$ and for $N=256$ the optimal value of compression factor is $\mu=21$, which gives the highest average value of SQNR. In this range of input signal variance the average values of SQNR is $39.9449 \mathrm{~dB}, 39.9608 \mathrm{~dB}, 39.9081 \mathrm{~dB}, 39.7166 \mathrm{~dB}$ for $\mu=18,21,28,40$, respectively. It can be observed that the average value of SQNR for $\mu=21$ is better than the values of average SQNR for the rest of analyzed values of compression factor $\mu$.

\section{CONCLUSION}

In this paper we formulated the new two-step method for optimizing compression factor $\mu$, which provides great accuracy in only two steps compared to the Muller's iterative method which gives optimal compression factor value in the $9^{\text {th }}$ or $10^{\text {th }}$ iteration, depending of number of quantization levels. The conclusion is that the new two-step method is much simpler and faster than Muller's iterative method. Since the new two-step method determines the optimal compression factor for unit signal variance, the second goal of the paper was to determine the optimal value of $\mu$ in the case when the input signal variance changes in the narrow range of input signal variance, $[-3 \mathrm{~dB}, 3 \mathrm{~dB}]$ and $[-4 \mathrm{~dB}, 2 \mathrm{~dB}]$. Based on the average value of SQNR, we concluded which value of compression factor is optimal for certain variance range. It has been shown that for the variance range $[-3 \mathrm{~dB}, 3 \mathrm{~dB}]$ compression factor value $\mu=18$ while for the variance range $[-4 \mathrm{~dB}, 2 \mathrm{~dB}]$ optimal is $\mu=21$. We also concluded that the optimal compression factor value does not depend only on the variance range width, but also on the variance range boundaries, because the optimal compression factor value is not the same for the considered ranges of variance. 
Acknowledgement: This paper was realized as a part of the projects TR 32035, financed by the Ministry of Education and Science of the Republic of Serbia within the framework of integrated and interdisciplinary research for the period 2011-2015.

\section{REFERENCES}

[1] N. S. Jayant and P. Noll, Digital coding of waveforms, Principles and Applications to Speech and Video, 2nd ed., New Jersey, Prentice Hall, Chapter 4, pp. 115-220, 1984.

[2] Na S., On the Support of Fixed-Rate Minimum Mean-Squared Error Scalar Quantizers for a Laplacian Source, IEEE Trans.Inf. Theory, 50 (2004), No. 5, 937-944.

[3] S. Na and D. L. Neuhoff, "Asymptotic MSE distortion of mismatched uniform scalar quantization", IEEE Trans. Inf. Theory, vol. 58, no. 5, pp. 3169-3181, May. 2012.

[4] HERRERO Rolando, INGLE Vinay, "Backward and forward linear prediction applied to ultraspectral image processing", Signal, Image and Video Processing, 2015, vol. 10, no. 4, p. 639-646. ISSN: $1863-$ 1711. DOI: $10.1007 / \mathrm{s} 11760-015-0788-\mathrm{y}$

[5] HERRERO Rolando, INGLE Vinay, "Ultraspectral image compression using two-stage prediction: Prediction gain and rate-distortion analysis”, Signal, Image and Video Processing, 2015, vol. 10, no. 4, p. 729-736. ISSN: 1863-1711. DOI: $10.1007 / \mathrm{s} 11760-015-0801-5$

[6] D. Aleksić, Z. Perić, J. Nikolić, „Support region determination of quasi-logarithmic quantizer for Laplacian source“, Przeglad Elektrotevhniczny, vol.88, no. 7A, pp. 130-132, 2012.

[7] Z. Perić, D. Aleksić, M. Stefanović, J. Nikolić, „New approach to support region determination of the $\mu-$ law quantizer", Electronica ir Electrotechnika, vol. 19, no. 8, pp.111-114, 2013

[8] Z. Perić, A. Jovanović, M. Tančić, „New Iterative Method for Optimization of Quasi-logarithmic Quantizer for Laplacian Source", presented at conference ICEST, June 25-27,2014, Vol. 1, str. 7-10, ISBN:978-86-6125-108-5

[9] J. Nikolić, Z. Perić, D. Aleksić, "Optimization of $\mu$-law companding quantizer for Laplacian source using Muller's method”, Przeglad Elektrotevhniczny, vol. 89, no. 3A, pp. 206-208,2013.

[10] Cheney W., Kincaid D., Numerical Mathematics and Computing, $6^{\text {th }}$ Edition, Thomson Higher Education, Belmont (2008)

[11] Atkinson K., An Introduction to Numerical Analysis, John Wiley \& Sons, New York (1989) 